\title{
Autism spectrum disorder in individuals with anorexia nervosa and in their first- and second-degree relatives: Danish nationwide register-based cohort-study
}

Susanne V. Koch, Janne T. Larsen, Svend E. Mouridsen, Mette Bentz, Liselotte Petersen, Cynthia Bulik, Preben B. Mortensen and Kerstin J. Plessen

\section{Background}

Clinical and population-based studies report increased prevalence of autism spectrum disorders (ASD) in individuals with anorexia nervosa and in their relatives. No nationwide study has yet been published on co-occurrence of these disorders.

\section{Aims}

To investigate comorbidity of ASD in individuals with anorexia nervosa, and aggregation of ASD and anorexia nervosa in their relatives.

\section{Method}

In Danish registers we identified all individuals born in 1981-2008, their parents, and full and half siblings, and linked them to data on hospital admissions for psychiatric disorders.

\section{Results}

Risk of comorbidity of ASD in probands with anorexia nervosa and aggregation of ASD in families of anorexia nervosa probands were increased. However, the risk of comorbid and familial ASD did not differ significantly from comorbid and familial major depression or any psychiatric disorder in anorexia nervosa probands.

\section{Conclusions}

We confirm aggregation of ASD in probands with anorexia nervosa and in their relatives; however, the relationship between anorexia nervosa and ASD appears to be nonspecific.

\section{Declaration of interest}

C.B. is a consultant for Shire Pharmaceuticals.

\section{Copyright and usage}

(c) The Royal College of Psychiatrists 2015.
The aetiology of anorexia nervosa is multifactorial and consists of a complex combination of genetic and environmental factors. Population-based twin studies estimate a heritability between 28 and $74 \% .{ }^{1-4}$ Anorexia nervosa is often associated with comorbid psychiatric disorders, such as depression, anxiety, obsessive-compulsive, personality and substance use disorders. ${ }^{1,5,6}$ Familial and population-based studies report co-occurrence of anorexia nervosa and autism spectrum disorders (ASD) in families $^{7}$ and an overrepresentation of ASD in individuals with eating disorders. ${ }^{8}$ Finally, in recent years a number of clinical and neuropsychological studies support the existence of shared traits between individuals with anorexia nervosa and ASD, including problems with central coherence, set-shifting and theory of mind in both disorders. ${ }^{9-14}$ These traits could present shared endophenotypes of anorexia nervosa and ASD, which characterise individuals or specific subgroups of individuals with anorexia nervosa; subgroups that may represent those who have a prolonged or chronic course of illness. ${ }^{5,14,15}$ Moreover, some authors have hypothesised that spectrum traits may manifest as anorexia nervosa in females and as ASD in males. ${ }^{10}$ Tailoring treatment for those subgroups of individuals with anorexia nervosa who either have comorbid ASD or subthreshold autism spectrum symptoms and traits might alter the course of anorexia nervosa in a favourable direction. ${ }^{16}$ It is therefore important to identify and to describe the co-occurrence of ASD in individuals with anorexia nervosa and in their families and vice versa.

Clinical and population-based studies describing the coexistence of anorexia nervosa and ASD in families or on the psychological traits of social cognition are, however, limited by small samples of predominantly females (Råstam 2003, $n=51$; Wentz 2005, $n=30) .^{6,8,17}$ Confirmatory evidence in large population-based samples does not yet exist. In Denmark we have access to data from public national registers with information on all people admitted to hospital for psychiatric disorders since 1969 onwards. ${ }^{18}$ The aim of this study was to investigate the co-occurrence of anorexia nervosa and ASD in probands and in their first- and second-degree relatives in a nationwide study. We hypothesised (a) that ASD would be overrepresented in probands with anorexia nervosa and vice versa (comorbidity) and (b) in their families (cross-diagnostic genetic liability) and (c) that we could identify a dose-response effect of the presence of ASD in these relatives according to the degree of shared genetic material as a measure for a common genetic aetiology for anorexia nervosa and ASD. Finally, we repeated all analyses for comorbid and familial major depression and any psychiatric disorder, to determine whether any observed associations were specific to anorexia nervosa and to ASD.

\section{Method}

\section{Study population}

We identified all individuals born in Denmark from 1 May 1981 to 31 December 2008 in the Danish Civil Registration System. ${ }^{19}$ The register includes the personal identification number, gender, date of birth, continuously updated information on vital status and parents' identification numbers. The identification number is used as a personal identifier in all national registers, enabling unique linkage between registers. We excluded those lacking a link to the mother and to information on parental ages at date of the child's birth $(n=11536$ (0.7\%) individuals, mostly lacking information on paternal age). 


\section{Assessment of anorexia nervosa, ASD and any mental disorder}

Individuals in the study population, their parents, and their full and half siblings were linked with the Danish Psychiatric Central Research Register, ${ }^{18}$ which registers all admissions to Danish psychiatric in-patient facilities since 1 April 1969, and with the Danish National Patient Register, ${ }^{20}$ which registers all admissions to somatic in-patient facilities since 1 January 1977. Since 1995 and onwards, the National Patient Register has also included psychiatric contacts in general hospitals, and both the National Patient Register and the Psychiatric Central Research Register have included information on out-patient contacts as well. Until 31 December 1993, the diagnostic system used was ICD- $8,{ }^{21}$ and since 1 January 1994, the diagnostic system ICD- $10^{22}$ has been used.

Given our aim to study comorbidity between anorexia nervosa and ASD in an individual, we included all members of the population classified as having anorexia nervosa or atypical anorexia (that is ICD-8 code $306.5 \mathrm{x}$ or ICD-10 code F50.0-F50.1) or infantile autism, atypical autism or Asperger syndrome (ICD-8 code 299.00-299.01 or ICD-10 code F84.0-F84.1, F84.5) if they had a record in the National Patient Register or Psychiatric Central Research Register of in- or out-patient care for that disorder. Cases of anorexia nervosa were not counted before the age of 9 years, because those diagnoses represented childhood eating disorders and not fully developed anorexia nervosa. ${ }^{23}$

In a second step, to study familial aggregation of the two disorders, we included index probands and their parents and siblings diagnosed with eating disorder (ICD-8 code 306.5 or ICD-10 code F50.0-F50.1, F50.8), ASD (ICD-8 code 299.00-299.01 or ICD-10 code F84.0-F84.1, F84.5, F84.8-F84.9), major depression (ICD-8 code $296.09,298.09$ or ICD-10 code F32, F33) or any psychiatric disorder (ICD-8 code $290-315$ or ICD-10 code F00-F99), if they had a record of in- or out-patient care for that disorder. The date of onset was defined as the first day of the first contact (in- or out-patient) leading to a diagnosis of that particular disorder.

\section{Study design}

A total of 1724189 people born in Denmark were followed up from date of birth until the first diagnosis with the disorder of interest, death, emigration from Denmark or 31 December 2012, whichever came first. The Danish Data Protection Agency has approved the study.

\section{Statistical analysis}

We estimated hazard ratios (HR; using Cox regression in Stata 12 for Windows) for both anorexia nervosa and ASD for probands, who had anorexia nervosa or ASD, respectively, or were exposed to a first- or second degree relative with one or the other of the disorders, to detect co-occurrence of anorexia nervosa and ASD independent of the sequence of the disorders. ${ }^{24}$ Moreover, we compared HRs for anorexia nervosa and ASD, respectively, in probands of families with major depression or any psychiatric disorder to distinguish between a specific effect of co-occurrence of the two disorders or a general psychiatric vulnerability. Analyses were adjusted for maternal and paternal age, for calendar time (to adjust for birth-cohort effect) as a time-dependent variable and for gender by means of separate underlying hazard functions. Calendar time was categorised as 1981-1984, 19851989, 1990-1992, 1993-1994, 1995-1999, 2000-2004, 2005-2010 and 2011-2012 taking into account the above mentioned changes in registration practice over time. Maternal age was categorised as less than 20 and 20 or older, whereas paternal age was categorised as less than 20, 20-39 and 40 or older according to how parental age acts as a risk factor for psychiatric illness in the offspring. ${ }^{25}$ Familial psychiatric history was coded as time-dependent variables for probands, first-degree relatives (mothers, fathers and full siblings) and second-degree relatives (maternal and paternal half siblings) separately. Individuals without second-degree relatives and those without registry-identified fathers were analysed separately. Furthermore, we performed supplementary analyses of gender and age at outcome-diagnosis stratified by age $<16$ and $\geqslant 16$ years of age (results not shown). We used 2-tailed chi-square test for descriptive statistics.

\section{Results}

In the study population of 1724189 individuals, we identified 5006 individuals (358 males, 4648 females; male:female ratio 1:13.0) who had a diagnosis of anorexia nervosa, and 12606 individuals (10012 males, 2594 females; male:female ratio 3.9:1) with a diagnosis of ASD (Table 1). In total, $45.8 \%$ of the females and $58.4 \%$ of the males were diagnosed with anorexia nervosa for the first time before the age of 16 years $(P<0.001)$. More females were given a first diagnosis of infantile or atypical autism $(64.4 \%$ v. $59.1 \%, P=0.009)$ and fewer a diagnosis of Asperger syndrome (35.6\% v. $40.9 \%, P<0.001$ ) before the age of 16 years compared with the males.

\section{Probands}

\section{Risk of ASD in probands with anorexia nervosa}

Probands with a first diagnosis of anorexia nervosa, had a highly elevated risk of receiving a second diagnosis of ASD $(\mathrm{HR}=15.08$, 95\% CI 12.23-18.58) (Table 2). This risk for males $(\mathrm{HR}=22.04$, 95\% CI 15.39-31.58) was higher than for females $(\mathrm{HR}=12.82$, 95\% CI 9.94-16.53). Males with anorexia nervosa had a higher risk of a late autism spectrum diagnosis ( $\geqslant 16$ years of age), whereas females with anorexia nervosa were equally diagnosed with ASD before and after the age of 16 years (results not shown). However, we found an even higher risk for being diagnosed with ASD in probands, whose first diagnosis was major depression $(\mathrm{HR}=34.76,95 \%$ CI 31.51-38.36).

\section{Risk of anorexia nervosa in probands with ASD}

Likewise, probands, who had ASD as their first diagnosis, had an elevated risk of having a second diagnosis of anorexia nervosa $(\mathrm{HR}=5.39,95 \%$ CI 4.37-6.64) (Table 2) with no differences with respect to gender or age at anorexia nervosa diagnosis (results not shown). The risk of developing anorexia nervosa, however, was even higher after an initial diagnosis of major depression $(\mathrm{HR}=17.67,95 \%$ CI 16.12-19.36).

\section{Family history}

\section{Risk of ASD with a family history of anorexia nervosa}

A family history of anorexia nervosa was associated with an elevated risk of receiving a diagnosis of ASD (Table 3). The HR for being diagnosed with ASD if a first-degree relative had anorexia nervosa was 1.80 (95\% CI 1.43-2.28) and the HR was 1.44 (95\% CI $0.94-2.21)$, if a second-degree relative had anorexia nervosa. However, these HRs were of the same magnitude as those seen in families with a history of major depression (HR for first-degree relatives 1.78 (95\% CI 1.65-1.92) and HR for second-degree relatives 1.37 (95\% CI 1.11-1.69)) and those in families with any psychiatric disorder (Table 3). We thus did not find a dose-response effect according to the degree of shared genetic material among relatives. 

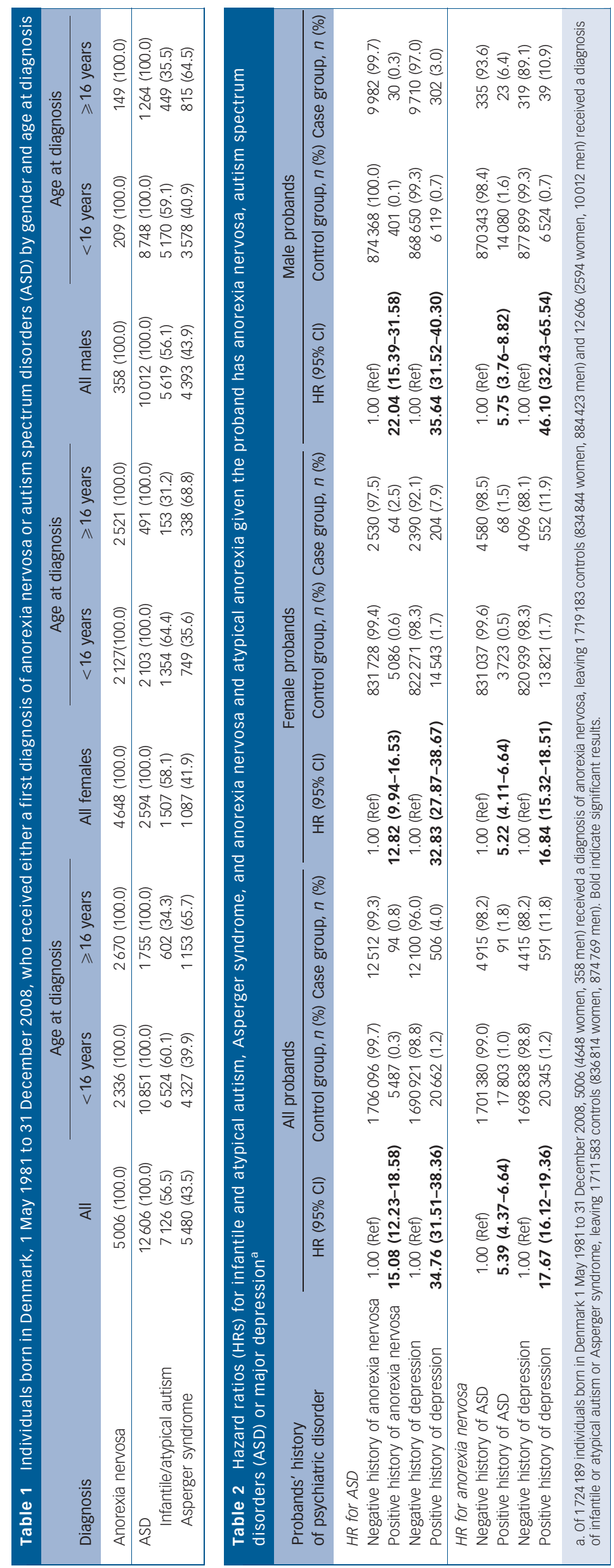

Risk of anorexia nervosa with a family history of ASD

A family history of ASD also increased the risk of being diagnosed with anorexia nervosa ( $\mathrm{HR}=1.45$ (95\% CI 1.09-1.94) for having a first-degree relative with ASD and HR $=1.40$ (95\% CI 0.89-2.20) for having a second-degree relative with ASD) (Table 4). Similar to the outcome of ASD, we did not observe a dose-response effect according to the degree of shared genetic material among relatives. Furthermore, a family history of ASD yielded a significantly lower risk for developing anorexia nervosa compared with families with a history of anorexia nervosa (Table 4). Moreover, we observed an equally elevated risk for developing anorexia nervosa among probands, who had a family member with major depression (HR for first-degree relatives 1.34 (95\% CI 1.19-1.51) and HR for second-degree relatives 1.02 (95\% CI $0.73-1.43)$ ). Families with a history of any psychiatric disorder showed a similar pattern.

\section{Familial aggregation of anorexia nervosa}

A family history of anorexia nervosa was a risk factor for developing anorexia nervosa with an HR of 3.92 (95\% CI 3.124.93) if a first-degree relative had the disorder and an HR of 1.83 (95\% CI, 1.01-3.32) if a second-degree relative had the disorder (Table 4). This represents a dose- response effect according to the degree of shared genetic material and underscores the role of genetic factors in the pathogenesis of anorexia nervosa. A subanalysis showed that especially anorexia nervosa in mothers and sisters contributed to the increased risk (both genders); however, we identified only a few cases of anorexia nervosa among fathers and brothers, which may have influenced the statistical power of the estimates (results not shown). Thus, we neither could detect any differential influences of a family history of anorexia nervosa on the proband according to gender; nor did probands with early diagnosis of anorexia nervosa seem to have a more pronounced aggregation of anorexia nervosa in the family.

\section{Familial aggregation of ASD}

Moreover, the risk for ASD was highly elevated when exposed to ASD in a first- (HR $=7.79,95 \%$ CI 7.15-8.50) and a seconddegree relative ( $\mathrm{HR}=2.97,95 \% \mathrm{CI} 2.47-3.57)$ (Table 3). Females were at highest risk (females $\mathrm{HR}=10.24$ (95\% CI 8.71-12.04) v. males $\mathrm{HR}=7.09$ (95\% CI 6.40-7.85)). ASD was most frequently present in fathers and brothers (results not shown), but ASD among the female members of the family also contributed to the results.

\section{Unspecific diagnoses}

In additional analyses, we included a broader phenotype (the unspecific diagnoses of the two categories in ICD-10, such as code F50.8 (eating disorders - NOS) and code F84.8+.9 (ASD - NOS), respectively) to explore the specificity of the findings, which did not alter the main results.

\section{Other effects}

Additional analyses could not confirm any specific effect of any of the measured psychiatric disorders in half-siblings (divided into maternal or paternal half-siblings), nor gender effects of full- or half-siblings. To exclude the possibility that unknown fathers could have influenced the analyses, we repeated the analyses restricted to families where all fathers were known, which also did not change the main findings. 


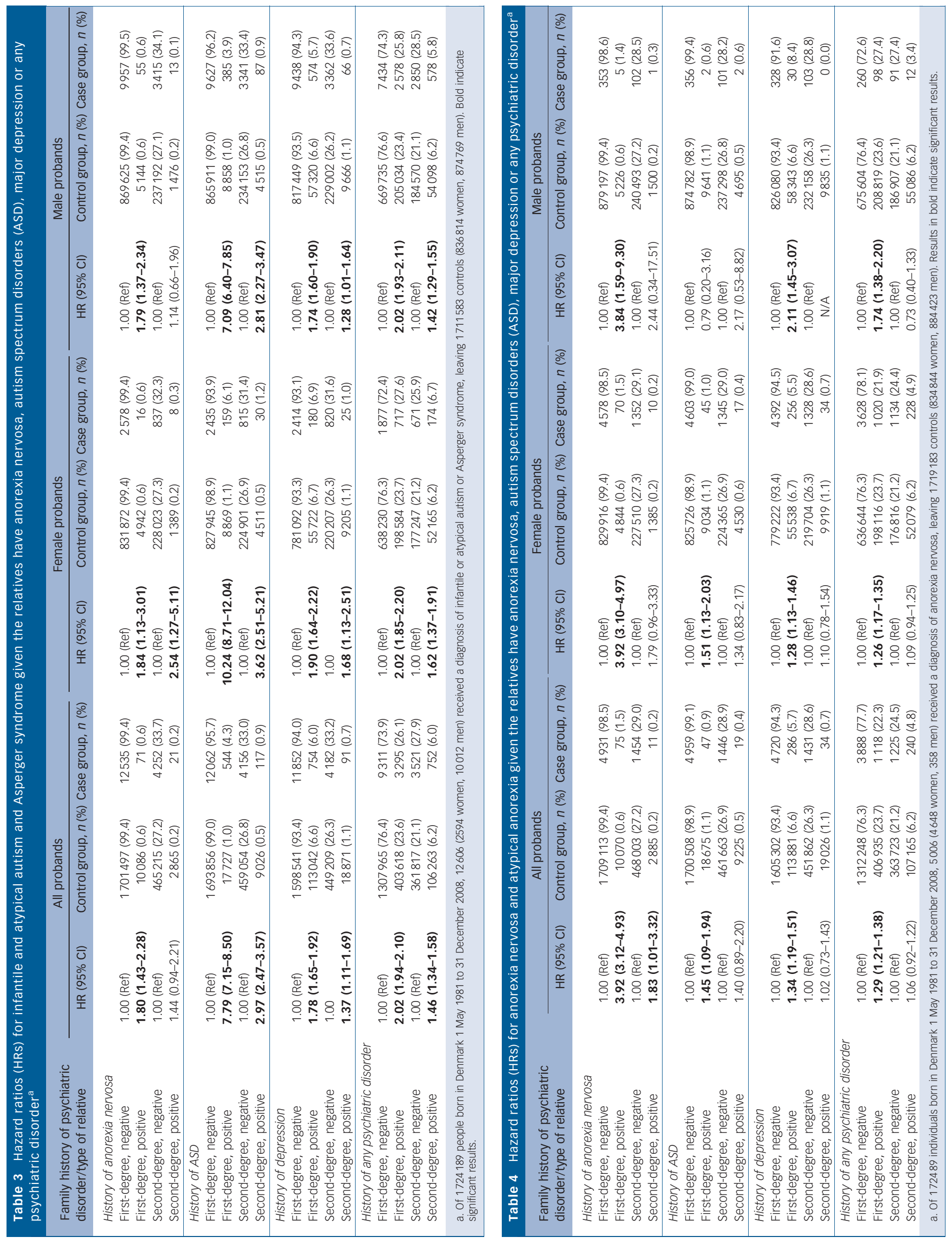




\section{Discussion}

\section{Risk of anorexia nervosa and co-occurring ASD}

We studied comorbidity and familial aggregation of anorexia nervosa and of ASD in a nationwide sample. This study corroborates earlier findings and confirms that individuals with anorexia nervosa have a higher risk of a co-occurring ASD, and that a family history of anorexia nervosa increases the risk for developing ASD. ${ }^{7,8}$ Similarly, individuals with ASD had an increased risk for a later diagnosis of anorexia nervosa and a family-member with ASD increased the risk of developing anorexia nervosa. However, the risk of developing anorexia nervosa was also increased in individuals with a diagnosis of major depression or any psychiatric disorder. Moreover, the risk was also increased if probands had family members with any of these psychiatric disorders. Furthermore, the study could not confirm a doseresponse effect of ASD in relatives of individuals with anorexia nervosa according to the degree of shared genetic material. Hence, our results did not support the hypothesis that ASD acts as a specific genetic or familial risk factor for developing anorexia nervosa, because the risk for developing anorexia nervosa was increased both by the presence of ASD in probands (comorbidity) or ASD in a family-member (familial aggregation) but also the presence of major depression or other psychiatric disorders increased this risk. Earlier observations of an augmented risk for co-occurring anorexia nervosa and ASD in families, thus, may express a non-specific genetic or familial vulnerability, which may also act as a risk factor for developing other psychiatric disorders.

\section{Familiality}

This study replicates earlier findings concerning familial aggregation $^{26}$ and heredity of anorexia nervosa, ${ }^{1-4}$ documenting a four times higher risk for developing anorexia nervosa in individuals with a first-degree relative with anorexia nervosa and a two times higher risk of the disorder with a second-degree relative. As expected, the dose-response effect in relatives with ASD is even stronger, which further confirms the heritability of ASD. ${ }^{27}$ These results support the good quality of the data.

\section{ASD as a non-specific risk factor for anorexia nervosa}

Several studies have demonstrated familial aggregation of major psychiatric disorders, ${ }^{28}$ including anorexia nervosa, ${ }^{26,29}$ in a cross-diagnostic perspective. Thus, familial aggregation of two or more disorders has been demonstrated for several psychiatric disorders, such as schizophrenia and bipolar disorder, ${ }^{30}$ Tourette syndrome and obsessive-compulsive disorder ${ }^{31}$ and anorexia nervosa and depression. ${ }^{1,32}$ Our findings, demonstrating that ASD is a non-specific familial risk factor for anorexia nervosa and vice versa, thus are in accordance with earlier findings suggesting that psychiatric disorders in general predispose to the development of different psychiatric disorders in the offspring. ${ }^{33}$ However, the specific co-occurrence of anorexia nervosa and ASD in families, has to our knowledge not been studied previously in a large population-based setting. Our results indicate that a simple Mendelian, genetic pathway does not explain the crossdiagnostic familial aggregation, and interactions must be more complex. ${ }^{34}$ The interaction between genetic and environmental factors may act through pleiotropy (i.e. the same genes may give rise to different disorders). Thus, a genome-wide association study has revealed pleiotropy in a number of psychiatric disorders, ${ }^{35}$ and suggests that pleiotropy act through identical genes that may be modulated by environmental factors to be expressed as various endophenotypes, ${ }^{36}$ which again might predispose for different psychiatric disorders.

\section{Comparison with findings from neuropsychological studies}

Neuropsychological studies, predominantly performed in adults, have reported the presence of social cognitive characteristics that are pathognomonic of ASD in individuals with anorexia nervosa and their relatives. ${ }^{6,8-14,17}$ These studies suggest that at least a subgroup of individuals with anorexia nervosa demonstrate social cognitive difficulties that may interfere with their ability to engage in family-based treatment, which is considered the treatment of choice for adolescents with anorexia nervosa. ${ }^{37,38}$ Most of these studies, however, find that the social cognitive deficits in patients with anorexia nervosa are less severe than in patients with ASD, reporting intermediate measures of social cognitive performance between patients with ASD and healthy controls. ${ }^{11,13}$ None of these studies has included other comparison groups such as patients and families with major depression or other psychiatric disorders. Thus, existing data cannot conclude whether the social cognitive deficits observed were specific to anorexia nervosa or would also be observed in individuals with other psychiatric disorders and in their family members. Although our results do not contradict the clinical observations; they argue for the inclusion of other pathological groups for comparison and determination of specificity in a cross-diagnostic perspective.

Other explanations for differences between our epidemiological findings and those of clinical studies of social cognition could be that these studies often consisted of small or selected samples and often included a combination of individuals with different eating disorders, i.e. also including those with bulimia nervosa, and individuals at different stages of the diseases. ${ }^{8,17}$ Finally, studies may not have accounted fully for the fact that social cognitive characteristics can be influenced by underweight or other neurobiological long-term effects of anorexia nervosa; thus, time to follow-up might have influenced the findings, because the knowledge about the impact of the duration of starvation on neuropsychological functions is limited. ${ }^{8,39}$ Moreover, many studies differ in their definition of full recovery of anorexia nervosa. ${ }^{40}$

\section{Gender and age at onset in relation to co-occurring anorexia nervosa and ASD}

Males were more frequently diagnosed with anorexia nervosa before the age of 16 years compared with females, but they did not more frequently have relatives with ASD or anorexia nervosa. The data presented here cannot explain this finding. This could simply point to the fact that males may be admitted to hospital treatment at younger ages than females. Two Nordic clinical studies have reported that males referred to hospital treatment were both diagnosed with anorexia nervosa at younger ages, had a shorter course of illness and had an overall more favourable prognosis than females. ${ }^{41,42}$

Males with anorexia nervosa also had a higher risk of having a second diagnosis of ASD, but the pattern of anorexia nervosa and ASD among their relatives did not indicate that genetic factors were the explanation for this. It might be that males with unrecognised ASD first receive a diagnosis when they have contact with the hospital system because of their eating problems. This is supported by the fact that the risk for developing ASD in the male group with anorexia nervosa was as high in the group of over 16-year-olds as in the younger age group, which is in contrast to 
the female group with anorexia nervosa, who more often were diagnosed with ASD before the age of 16 years.

Overall, this study cannot conclude that groups defined by clinical characteristics such as gender or early-diagnosis anorexia nervosa have a specifically increased risk for having ASD in the family, or vice versa. We are unable to determine from this study whether anorexia nervosa and ASD are alternative gender-specific expressions of the same genetic factor.

\section{Strengths and limitations}

We have included data from the total Danish population, which affords considerable statistical power, generalisability and a minimum of selection and recall bias. Hospital treatment is free of charge in Denmark, which makes it accessible for all socioeconomic groups. The Danish Civil Registration System does not allow people to be lost to follow-up and data are collected prospectively for public administrative purposes and not for this specific study. Further strengths are the large number of cases, in particular in males, and exposed family members and the unique linkage to family members across two generations.

Our study also has limitations. One is that we were only considering categorical diagnoses rather than more richly characterised dimensional characteristics, which may be more suitable for describing cross-diagnostic traits or shared endophenotypes or subphenotypes. Hence, we were unable to include data on subthreshold anorectic or autistic symptoms, or on psychological traits concerning social cognitive deficits that may influence the development of anorexia nervosa and ASD, or on patients with an intermediate phenotype between anorexia nervosa and ASD. These kinds of data are not available from the registers where only patients who reach the full diagnostic criteria (for example infantile autism) are registered. For anorexia nervosa and bulimia nervosa it is furthermore assumed that more than $50 \%$ of individuals with mild to moderate disorders are never admitted for hospital care, ${ }^{43}$ and therefore cannot be identified via the registers.

Eating disorder diagnoses in the Danish Registries are not yet systematically validated. Register diagnoses of schizophrenia, ${ }^{44}$ childhood autism ${ }^{45}$ and depression ${ }^{46}$ have, however, been validated and have shown good validity, and thus, diagnoses in the registries are deemed of good quality. One study has suggested though that comorbid diagnoses have less good coverage compared with the primary diagnoses, ${ }^{47}$ potentially leading to a number of missing comorbid diagnoses, which may have contributed to underestimation of the co-occurrence of anorexia nervosa and ASD.

Even though a small percentage of fathers were not identified at the child's birth and therefore hindered some linkages to family members, analysis restricted to probands, whose fathers were known, did not change the results; thus, this problem had a minor impact on data quality. We decided to also include ICD-8 diagnoses, to allow a larger population through the inclusion of older birth cohorts and mental health diagnoses of parents, even though this system generated more incomplete and imprecise information, compared with ICD-10 diagnoses. Thus, inferior recognition of psychiatric disorders in parents may have contributed to lower statistical power in some of the subgroup analyses.

\section{Summary}

Our results suggest that an individual who has already developed anorexia nervosa is also at increased risk for being diagnosed with ASD or vice versa. Further, we observe an aggregation of anorexia nervosa and ASD in families. The risk for developing anorexia nervosa, however, is equally increased if the individual has depression or any other psychiatric disorder, or if these disorders are present in the family. The present data, thus, do not support a specific genetic relationship between anorexia nervosa and ASD; however, having any psychiatric disorder or having relatives with any psychiatric disorder is a non-specific risk factor for developing anorexia nervosa. Thus, our findings are in line with recent studies that point to overlapping genetic liabilities for several neuropsychiatric disorders. The risk of developing anorexia nervosa showed a dose-response effect according to having first- and second-degree relatives with anorexia nervosa and supports the heritability of anorexia nervosa; likewise the heritability of ASD is confirmed. Having family members with anorexia nervosa or ASD did not seem to impose a specific risk on males to develop anorexia nervosa compared with females or in relation to early onset of anorexia nervosa.

\section{Implications}

Our results indicate that the presences of a psychiatric disorder may act as a non-specific risk factor for the development of other psychiatric disorder in the offspring, which further extends our knowledge concerning the risk of mental health problems in children of parents with psychiatric disorders. Earlier literature has focused mostly on children of parents with affective disorders or psychosis, ${ }^{28}$ but children with parents with anorexia nervosa are also at elevated risk. ${ }^{32}$ Moreover, this study emphasises the importance of dimensional and cross-diagnostic approaches to advance our understanding of the characteristics of social cognitive functioning in individuals with anorexia nervosa, especially when distinguishing whether these represent state or trait characteristics and whether these putative endophenotypes are of prognostic value. Furthermore, it is important to describe co-occurrence and sequential comorbidity with other psychiatric symptoms or disorders in anorexia nervosa illness trajectory, and to be aware of the presentation of early signs and manifestations of the disorder that may differ somewhat between the genders. Similarly, future studies should further target the possible aetiology and impact of shared cross-diagnostic genetic liabilities, preferably in longitudinal studies. A more thorough assessment of neuropsychological characteristics and recognition of co-occurrence of psychiatric disorders will hopefully lead to individually tailored treatment-courses for individuals with anorexia nervosa, which in turn may lead to a shorter course of the illness and improved outcome.

\footnotetext{
Susanne v. Koch, MD, PhD, Mental Health Centre for Child and Adolescent Psychiatry, Copenhagen Region and Institute of Clinical Medicine, Faculty of Health Sciences, University of Copenhagen, Copenhagen, Denmark; Janne T. Larsen, MSC, National Center for Register-based Research, School of Business and Social Sciences, University of Aarhus, Aarhus, and The Lundbeck Foundation Initiative for Integrative Psychiatric Research, iPsych, Department of Biomedicine, Faculty of Health, Aarhus University, Denmark; Svend E. Mouridsen, MSCPsych, Mette Bentz, MSCPsych, Mental Health Centre for Child and Adolescent Psychiatry, Copenhagen Region, Denmark; Liselotte Petersen, MSc, PhD, National Center for Register-based Research, School of Business and Social Sciences, University of Aarhus, Aarhus, iPsych, Department of Biomedicine, Faculty of Health, Aarhus University, and The Lundbeck Foundation Initiative for Integrative Psychiatric Research, Aarhus, Denmark Cynthia Bulik, PhD, FAED, Department of Psychiatry, University of North Carolina, Chapel Hill, North Carolina, USA, and Department of Medical Epidemiology and Biostatistics, Karolinska Institutet, Stockholm, Sweden; Preben B. Mortensen, MD, DMSci, National Center for Register-based Research, School of Business and Social Sciences, University of Aarhus, Aarhus, and The Lundbeck Foundation Initiative for Integrative Psychiatric Research, iPsych, Department of Biomedicine, Faculty of Health, Aarhus University, Denmark; Kerstin J. Plessen, MD, PhD, Mental Health Health, Aarhus University, Denmark; Kerstin J. Plessen, MD, PhD, Mental Health
Centre for Child and Adolescent Psychiatry, Copenhagen Region and Institute of Clinical Medicine, Faculty of Health Sciences, University of Copenhagen, Copenhagen,
} Denmark

Correspondence: Susanne V. Koch, Mental Health Centre for Child and Adolescent Psychiatry, University of Copenhagen, Lersø Parkallé 107, Ith, DK 2100, Copenhagen, Denmark. Email: Susanne.vinkel.koch@regionh.dk

First received 23 Jun 2014, accepted 21 Aug 2014 


\section{Funding}

Mental Health Services, Capital Region, Copenhagen, Denmark.

\section{References}

1 Wade TD, Bulik CM, Neale M, Kendler KS. Anorexia nervosa and major depression: shared genetic and environmental risk factors. Am J Psychiatry 2000; 157: 469-71.

2 Kortegaard LS, Hoerder K, Joergensen J, Gillberg C, Kyvik KO. A preliminary population-based twin study of self-reported eating disorder. Psychol Med 2001; 31: 361-5.

3 Trace SE, Baker JH, Penas-Lledo E, Bulik CM. The genetics of eating disorders. Annu Rev Clin Psychol 2013; 9: 589-620.

4 Rastam M, Taljemark J, Tajnia A, Lundstrom S, Gustafsson P, Lichtenstein P, et al. Eating problems and overlap with ADHD and autism spectrum disorders in a nationwide twin study of 9- and 12-year-old children. ScientificWorldJournal 2013; 2013: 315429.

5 Steinhausen $\mathrm{HC}$. The outcome of anorexia nervosa in the 20th century Am J Psychiatry 2002; 159: 1284-93.

6 Rastam M, Gillberg C, Wentz E. Outcome of teenage-onset anorexia nervosa in a Swedish community-based sample. Eur Child Adolesc Psychiatry 2003; 12 (suppl 1): 178-90.

7 Gillberg C. Are autism and anorexia nervosa related? Nord J Psychiatry 1985 39: 307-12.

8 Huke V, Turk J, Saeidi S, Kent A, Morgan JF. Autism spectrum disorders in eating disorder populations: a systematic review. Eur Eat Disord Rev 2013; 21: 345-51.

9 Treasure J. Coherence and other autistic spectrum traits and eating disorders: building from mechanism to treatment. The Birgit Olsson lecture. Nord J Psychiatry 2013; 67: 38-42.

10 Zucker NL, Losh M, Bulik CM, LaBar KS, Piven J, Pelphrey KA. Anorexia nervosa and autism spectrum disorders: guided investigation of social cognitive endophenotypes. Psychol Bull 2007; 133: 976-1006.

11 Baron-Cohen S, Jaffa T, Davies S, Auyeung B, Allison C, wheelwright S. Do girls with anorexia nervosa have elevated autistic traits? Mol Autism 2013; 4: 24.

12 Tchanturia K, Smith E, Weineck F, Fidanboylu E, Kern N, Treasure J, et al. Exploring autistic traits in anorexia: a clinical study. Mol Autism 2013; 4: 44

13 Courty A, Maria AS, Lalanne C, Ringuenet D, Vindreau C, Chevallier C, et al. Levels of autistic traits in anorexia nervosa: a comparative psychometric study. BMC Psychiatry 2013; 13: 222.

14 Gillberg IC, Billstedt E, Wentz E, Anckarsater H, Rastam M, Gillberg C. Attention, executive functions, and mentalizing in anorexia nervosa eighteen years after onset of eating disorder. J Clin Exp Neuropsychol 2010; 32: 358-65.

15 Anckarsater H, Hofvander B, Billstedt E, Gillberg IC, Gillberg C, Wentz E, et al. The sociocommunicative deficit subgroup in anorexia nervosa: autism spectrum disorders and neurocognition in a community-based, longitudinal study. Psychol Med 2012; 42: 1957-67.

16 Huke V, Turk J, Saeidi S, Kent A, Morgan JF. The clinical implications of high levels of autism spectrum disorder features in anorexia nervosa: a pilot study. Eur Eat Disord Rev 2014; 4: 116-21.

17 Wentz E, Lacey JH, Waller G, Rastam M, Turk J, Gillberg C. Childhood onset neuropsychiatric disorders in adult eating disorder patients. A pilot study. Eur Child Adolesc Psychiatry 2005; 14: 431-7.

18 Mors O, Perto GP, Mortensen PB. The Danish Psychiatric Central Research Register. Scand J Public Health 2011; 39: 54-7.

19 Pedersen CB. The Danish Civil Registration system. Scand J Public Health 2011; 39: 22-5.

20 Lynge E, Sandegaard JL, Rebolj M. The Danish National Patient Register. Scand J Public Health 2011; 39: 30-3.

21 World Health Organization. Classification of Diseases: Extended Danish-Latin Version of the World Health Organization International Classification of Diseases, 8th Revision, 1965. Danish Board of Health, 1971.

22 World Health Organization. The IDC-10 Classification of Mental and Behavioural Disorders: Diagnostic Criteria for Research. World Health Organization, 1993.

23 Micali N, Simonoff E, Elberling H, Rask CU, Olsen EM, Skovgaard AM. Eating patterns in a population-based sample of children aged 5 to 7 years: association with psychopathology and parentally perceived impairment. J Dev Behav Pediatr 2011; 32: 572-80.

24 Schnack TH, Zdravkovic S, Myrup C, Westergaard T, Wohlfahrt J, Melbye M. Familial aggregation of cryptorchidism-a nationwide cohort study. Am J Epidemiol 2008; 167: 1453-7.

25 McGrath JJ, Petersen L, Agerbo E, Mors O, Mortensen PB, Pedersen CB. A comprehensive assessment of parental age and psychiatric disorders. JAMA Psychiatry 2014; 71: 301-9.

26 Steinhausen $\mathrm{HC}$, Jakobsen $\mathrm{H}$, Helenius $\mathrm{D}$, Munk-Jorgensen $\mathrm{P}$, Strober M. A nation-wide study of the family aggregation and risk factors in anorexia nervosa over three generations. Int J Eat Disord 2014; April 28 (Epub ahead of print).

27 Rutter ML. Progress in understanding autism: 2007-2010. J Autism Dev Disord 2011; 41: 395-404.

28 Steinhausen HC, Foldager L, Perto G, Munk-Jorgensen P. Family aggregation of mental disorders in the nationwide Danish three generation study. Eur Arch Psychiatry Clin Neurosci 2009; 259: 270-7.

29 Lindberg L, Hjern A. Risk factors for anorexia nervosa: a national cohort study. Int J Eat Disord 2003; 34: 397-408.

30 Lichtenstein P, Yip BH, Bjork C, Pawitan Y, Cannon TD, Sullivan PF, et al. Common genetic determinants of schizophrenia and bipolar disorder in Swedish families: a population-based study. Lancet 2009; 373: 234-9.

31 do Rosario-Campos MC, Leckman JF, Curi M, Quatrano S, Katsovitch L, Miguel EC, et al. A family study of early-onset obsessive-compulsive disorder. Am J Med Genet B Neuropsychiatr Genet 2005; 136B: 92-7.

32 Fairburn CG, Cooper Z, Doll HA, Welch SL. Risk factors for anorexia nervosa: three integrated case-control comparisons. Arch Gen Psychiatry 1999; 56: 468-76.

33 Rasic $D$, Hajek T, Alda M, Uher R. Risk of mental illness in offspring of parents with schizophrenia, bipolar disorder, and major depressive disorder: a meta-analysis of family high-risk studies. Schizophr Bull 2014; 40: 28-38.

34 Uher R. Gene-environment interactions in severe mental illness. Front Psychiatry 2014; 5: 48

35 Smoller JW, Craddock N, Kendler K, Lee PH, Neale BM, Nurnberger J, et al. Identification of risk loci with shared effects on five major psychiatric disorders: a genome-wide analysis. Lancet 2013; 381: 1371-9.

36 Serretti A, Fabbri C. Shared genetics among major psychiatric disorders. Lancet 2013; 381: 1339-41.

37 Lock J, Le GD, Agras WS, Moye A, Bryson SW, Jo B. Randomized clinical trial comparing family-based treatment with adolescent-focused individual therapy for adolescents with anorexia nervosa. Arch Gen Psychiatry 2010; 67: 1025-32.

38 Treasure J, Claudino AM, Zucker N. Eating disorders. Lancet 2010; 375: 583-93.

39 Oldershaw A, Hambrook D, Tchanturia K, Treasure J, Schmidt U. Emotional theory of mind and emotional awareness in recovered anorexia nervosa patients. Psychosom Med 2010; 72: 73-9.

40 Couturier J, Lock J. What is remission in adolescent anorexia nervosa? A review of various conceptualizations and quantitative analysis. Int J Eat Disord 2006; 39: 175-83.

41 Lindblad F, Lindberg L, Hjern A. Anorexia nervosa in young men: a cohort study. Int J Eat Disord 2006; 39: 662-6.

42 Stoving RK, Andries A, Brixen K, Bilenberg N, Horder K. Gender differences in outcome of eating disorders: a retrospective cohort study. Psychiatry Res 2011; 186: 362-6.

43 Pagsberg AK, Wang AR. Epidemiology of anorexia nervosa and bulimia nervosa in Bornholm County, Denmark, 1970-1989. Acta Psychiatr Scand 1994; 90: 259-65.

44 Uggerby $\mathrm{P}$, Ostergaard SD, Roge R, Correll CU, Nielsen J. The validity of the schizophrenia diagnosis in the Danish Psychiatric Central Research Register is good. Dan Med J 2013; 60: A4578.

45 Lauritsen $M B$, Jorgensen $M$, Madsen KM, Lemcke S, Toft S, Grove J, et al. Validity of childhood autism in the Danish Psychiatric Central Register: findings from a cohort sample born 1990-1999. J Autism Dev Disord 2010; 40: 139-48.

46 Bock C, Bukh JD, Vinberg M, Gether U, Kessing LV. Validity of the diagnosis of a single depressive episode in a case register. Clin Pract Epidemiol Ment Health 2009; 5: 4.

47 Kessing LV. Gender differences in subtypes of late-onset depression and mania. Int Psychogeriatr 2006; 18: 727-38. 